\title{
Variações anatômicase sinusopatias: estudo por tomografia computadorizada
}

\section{Anatomical variations and sinusitis: a computed tomographic study}

\author{
Marcio M. Kinsui ${ }^{1}$, Arnaldo Guilherme', \\ Hélio K. Yamashita ${ }^{3}$
}

Palavras-chave: anatomia, cavidade nasal, turbinados, seios paranasais, tomografia computadorizada por raios $x$.

Key words: anatomy, nasal cavity, turbinates, paranasal sinuses, tomography.

\section{Resumo / Summary}

ntrodução: A tomografia computadorizada tem sido cada vez mais utilizada para a identificação e avaliação das variações anatômicas das cavidades nasais que podem levar às sinusites. Objetivo: Avaliar, por meio da tomografia computadorizada, a incidência de espessamento mucoso nos seios paranasais de pacientes com sintomas de sinusite e a correlação entre sinusopatias e presença de célula de Haller, concha média bolhosa e desvio de septo nasal localizado no meato médio. Forma de Estudo: Clínico retrospectivo. Material e Método: Foram avaliadas retrospectivamente 150 tomografias computadorizadas de seios paranasais de pacientes com idade igual ou superior a 13 anos, realizadas no Departamento de Diagnóstico por Imagem da Universidade Federal de São Paulo - Escola Paulista de Medicina, no período de julho de 1999 a outubro de 2001. Foram excluídos os exames de pacientes com pós-operatório de cirurgias de seios paranasais e de base de crânio, além dos portadores de lesões tumorais envolvendo estas regiões. Resultados: $70 \%$ dos pacientes apresentavam espessamento mucoso em pelo menos um dos seios paranasais. Observou-se a freqüência de $52,7 \%$ de sinusopatia maxilar, $28,0 \%$ de etmoidal, $13,0 \%$ de esfenoidal e $8,3 \%$ de frontal. Concha média bolhosa foi encontrada em $33,3 \%$ das cavidades nasais, desvio de septo nasal localizado no meato médio em 23,3\% e célula de Haller em 9,3\%. Conclusões: Os seios paranasais mais freqüentemente acometidos foram, em ordem decrescente: maxilar, etmoidal, esfenoidal e frontal. Não foi observado correlação entre sinusopatias e presença de célula de Haller, concha média bolhosa e desvio de septo nasal localizado no meato médio. ntroduction: Computed tomography has been increasingly used both to identify and to evaluate anatomy variations of nasal cavities that can lead to the development of sinusitis. Purpose: The purpose of the present study is to determine the incidence of mucosal abnormalities in paranasal sinuses found in CT scans of patients with symptons of sinusitis and analyze the correlation between sinusitis and presence of Haller's cell, concha bullosa and nasal septal deviation located in middle meatus. Study Design: Clinical retrospective. Material and Method: Paranasal sinus CT scans were obtained in 150 patients aged 13 years or more, from July 1999 to October 2001. The CT scans were performed in the Department of Radiology of Universidade Federal de São Paulo - Escola Paulista de Medicina. Patients with history of skull base or sinus surgery and tumor in these regions were excluded. Results: $70 \%$ of patients present mucosal abnormalities at least in one paranasal sinus. Maxillary sinusitis were observed in $52,7 \%$ of sinus, ethmoidal sinusitis in 28,0\%, sphenoidal sinusitis in $13,0 \%$ and frontal sinusitis in $8,3 \%$. Concha bullosa was observed in $33,3 \%$ of nasal cavities, nasal septal deviation (located in middle meatus) in $23,3 \%$ and Haller's cell in $9,3 \%$. Conclusions: The most affected paranasal sinuses were: maxillary, ethmoid, sphenoid and frontal. Correlation between sinusitis and presence of Haller's cell, concha bullosa and nasal septal deviation (located in middle meatus) was not observed.

\footnotetext{
1Pós-graduando do Departamento de O torrinolaringologia e Dístúrbios da Comunicação Humana da Universidade Federal de São Paulo - Escola Paulista de Medicina 2Professor Adjunto da Disciplina de O torrinolaringologia do Departamento de O torrinolaringologia e Distúrbios da Comunicação Humana da Universidade Federal de São Paulo - Escola Paulista de Medicina

${ }^{3}$ Professor Adjunto do Departamento de Diagnóstico por Imagem da Universidade Federal de São Paulo - Escola Paulista de Medicina

Trabalho realizado na Universidade Federal de São Paulo - Escola Paulista de Medicina Suporte financeiro: CNPq

Endereço para correspondência: Marcio Massao Kinsui - Rua Primitiva Vianco, 244, sala 1010 - 06016-901 0 sasco /SP -

Tel: (0xx11) 3654-3610 - Fax: (0xx11) 3681-9111 - E-mail: marciokinsui@hotmail.com

Artigo recebido em 30 de julho de 2002. Artigo aceito em 15 de agosto de 2002.
} 


\section{INTRODUÇÃO}

A compreensão exata da anatomia da cavidade nasal, com ênfase à parede lateral do nariz e suas variações, é de extrema importância para a cirurgia endoscópica naso-sinusal. As variações anatômicas que têm sido consideradas como predisponentes às sinusites incluem a concha média bolhosa, o desvio ou esporão do septo nasal, a bolha etmoidal hiperpneumatizada, a concha média paradoxal, o desvio medial ou lateral do processo uncinado, entre outras. A concha média bolhosa parece ser a mais freqüente das variações encontradas ${ }^{1}$.

0 desenvolvimento de novos instrumentos endoscópicos e técnicas associadas permitiu um novo méto do cirúrgico para o tratamento das sinusites crônicas, que visa a restauração da fisiologia normal, conseguida por meio do restabelecimento da drenagem mucociliar e da ventilação dos seios paranasais $s^{2,3,4}$. Em vista disso, uma entidade anatômica denominada complexo óstio-meatal tornou-se de suma importância, tanto no estudo dos fenômenos fisiopatológicos, como na tática cirúrgica.

O complexo óstio-meatal é composto pelo óstio do seio maxilar, infundíbulo etmoidal, processo uncinado, bolha etmoidal, hiato semilunar, recesso frontal, meato médio e concha média ${ }^{5}$. As variações anatômicas do complexo óstio-meatal têm sido implicadas na etiologia das sinusites crônicas e recorrentes e, por esta razão, a tomografia computadorizada de seios paranasais, em especial os cortes coronais, tem sido rotineiramente utilizada na avaliação dos pacientes com sintomas sinusais ${ }^{2,5}$. Deve-se ter em mente que, na maioria das vezes, a tomografia computadorizada é importante, não apenas para o diagnóstico da doença inflamatória em si, mas também para a visibilização das alterações anatômicas, tornando-se um excelente exame para o planejamento cirúrgico.

Com o uso rotineiro da tomografia computadorizada, observamos melhora na identificação das estruturas e das variações anatômicas das cavidades nasais e paranasais, entre elas, das células de Haller. Descritas inicialmente por Von Haller em 1803, estas células são consideradas expansões da pneumatização do complexo etmoidal localizadas inferiormente à bolha etmoidal e ao longo da porção medial do assoalho da órbita, relacionadas com o teto do seio maxilar. As primeiras observações foram realizadas através de dissecções de cadáveres, sendo até há pouco tempo dada pouca relevância a estas estruturas. A compreensão da fisiologia da drenagem mucociliar dos seios paranasais e 0 surgimento da tomografia computadorizada levantou a hipótese das células de $\mathrm{Haller}$ estarem associadas a rinossinusites, devido à sua localização junto ao infundíbulo etmoidal.

Este trabalho tem como objetivo avaliar, por meio da tomografia computadorizada, a incidência de espessamento mucoso dos seios maxilares, etmoidais, frontais e esfenoidais em pacientes com sintomas de sinusite e a ocorrência de células de Haller, concha média bolhosa e desvio de sep to nasal localizado no meato médio e suas correlações com sinusopatias maxilar, etmoidal e frontal.

\section{MATERIAL E MÉTODO}

Para este estudo foram avaliadas retrospectivamente 150 to mografias computadorizadas de seios paranasais de indivíduos de ambos os sexos, realizadas no Departamento de Diagnóstico por Imagem da Universidade Federal de São Paulo - Escola Paulista de Medicina, no período de julho de 1999 a outubro de 2001. Os exames incluídos na amostra apresentavam diagnóstico compatível com sinusopatia inflamatória ou de normalidade. Foram excluídos os exames de pacientes com pós-operatório de cirurgias de seios paranasais e de base de crânio, além dos portadores de lesões tumorais envolvendo estas regiões. Crianças com idade inferior a 13 anos também foram excluídas, pois os seios maxilares adquirem a forma adulta em torno desta idade, estabilizando suas dimensões próximo aos 18 anos, e os seios frontais e esfenoidais tornam-se viáveis à identificação radiológica por volta dos 4 anos de idade ${ }^{6}$. Além disso, abaixo dos 13 anos de idade, é bem maior a ocorrência de hipertrofia das tonsilas faríngeas, fator importante na gênese de sinusopatias em crianças e que influiria na avaliação da correlação das variações anatômicas da cavidade nasal com o comprometimento dos seios paranasais.

Os exames tomográficos foram realizados com os equipamentos Philips Secura e Philips Tomoscan AV. 0 protocolo dos exames incluiu a aquisição de imagens nos planos coronais, perpendiculares ao palato duro, do seio frontal (no limite anterior) ao seio esfenoidal (no limite posterior), com os pacientes posicionados em decúbito dorsal e hiperextensão cervical, utilizando o apoio de cabeça aprop riado para esta posição, e cortes de $3,0 \mathrm{~mm} / 3,0 \mathrm{~mm}$ de espessura/ incremento - com filtro de alta resolução. As imagens adquiridas no plano axial foram paralelas ao palato duro, desta estrutura (no limite inferior) ao seio frontal (no limite superior), com os pacientes em decúbito dorsal e posição cervical neutra, com apoio de cabeça também apropriado, e cortes de 3,0 $\mathrm{mm} / 3,0 \mathrm{~mm}$ de espessura/ incremento. As imagens foram adquiridas com 120/ $125 \mathrm{kV}$ e 224/ $225 \mathrm{mAs}$. As janelas tomográficas utilizadas foram 1500 - 2000/ 200 - 300 para partes ósseas e de 300 - 400/ 30 - 40 (largura/ nível) para partes moles.

O s exames foram analisados quanto à presença ou ausência de sinusopatia dos seios maxilares, etmoidais, frontais e esfenoidais. No presente trabalho, foi adotado o conceito clássico de Som, que considera patológica qualquer área demonstrável de mucosa, sendo classificada, então, como sinusopatia?.

As estruturas anatômicas estudadas foram a concha média, as células de Haller, os seios maxilares, etmoidais, frontais e esfenoidais, e o septo nasal (Figuras 1, 2 e 3). 
A concha média foi inicialmente avaliada, sendo classificada como bolhosa ao apresentar qualquer pneumatização. Posteriormente, foi estudada a distribuição das conchas médias bolhosas nos graus de sinusopatia.

Foi verificada a presença ou ausência de células de Haller, e sua distribuição nos diferentes graus de sinusopatia. Estas células foram definidas como uma pneumatização no rebordo orbitário ínfero-medial, delimitando o limite súperolateral do infundíbulo etmoidal, ao invés da bolha etmoidal, que o faz habitualmente.

Foi também registrada a presença ou ausência de desvio do septo nasal na região do meato médio e sua distribuição nos graus de sinusopatia, não sendo considerados desvios em outras regiões da cavidade nasal.

Para o estudo da correlação entre as estruturas analisadas (concha média bolhosa, célula de Haller e desvio do septo nasal) e os graus de sinusopatia, foi utilizado 0 teste do Qui-quadrado, com nível de significância de $5 \%$.

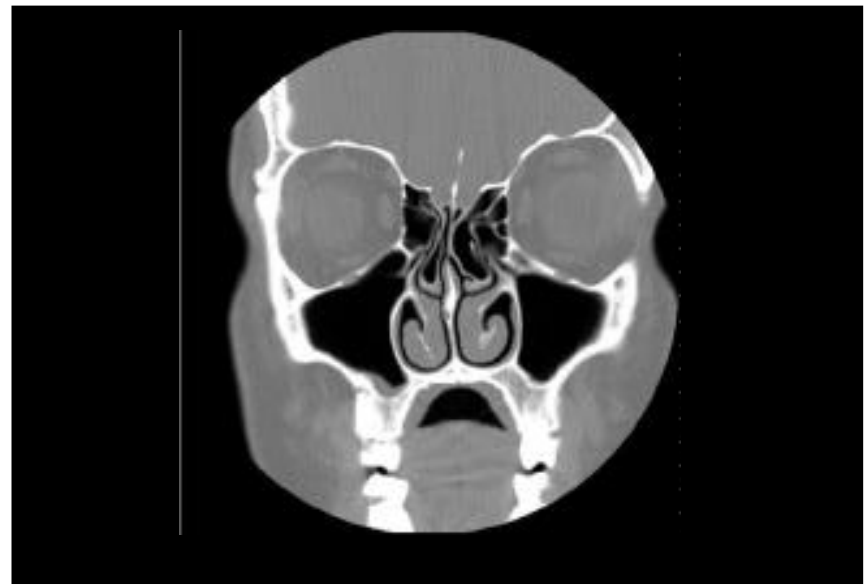

Figura 1. Corte coronal com concha média bolhosa bilateral

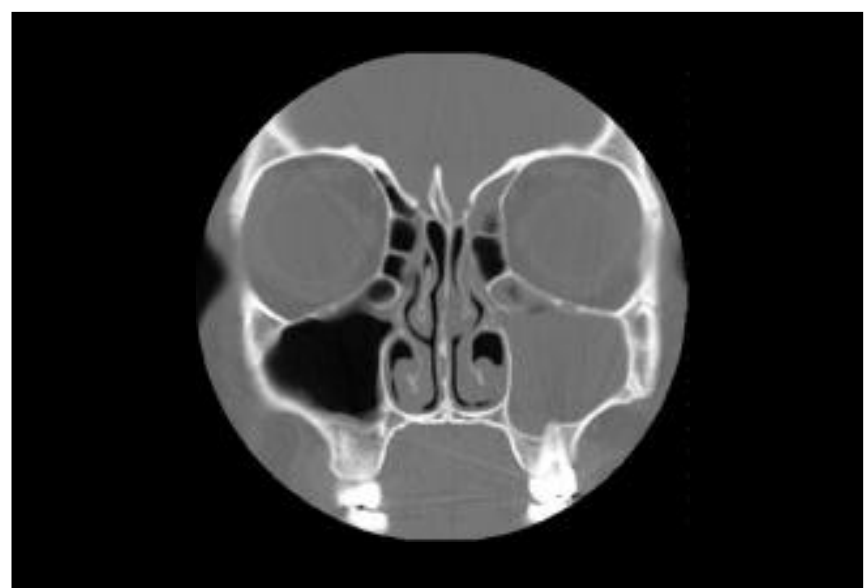

Figura 2. Corte coronal com célula de Haller bilateral (velada à esquerda) e sinusopatia maxilo-etmoidal à esquerda

\section{RESULTADOS}

A amostra foi composta por $85(56,7 \%)$ indivíduos do sexo feminino e 65 (43,3\%) do sexo masculino. A idade variou entre 13 e 98 anos (idade média de 38,2 anos com desvio padrão de 17,5 anos).

No Gráfico 1, observa-se sinusopatia em $70 \%$ dos pacientes. A distribuição dos seios paranasais mais acometidos encontra-se no Gráfico 2. A análise dos exames de tomografia computadorizada (Gráfico 3) revela que a concha média bolhosa é a alteração anatômica estudada mais freqüentemente encontrada, seguida pelo desvio de septo nasal com localização no meato médio e pela célula de Haller.

O bserva-se que não há relação significante entre a presença de célula Haller e sinuso patia maxilar, etmoidal e frontal (Tabelas 1, 2 e 3). Nas Tabelas 4, 5 e 6, não se observa relação significante entre a presença de concha média bolhosa e sinusopatia maxilar, etmoidal e frontal. As

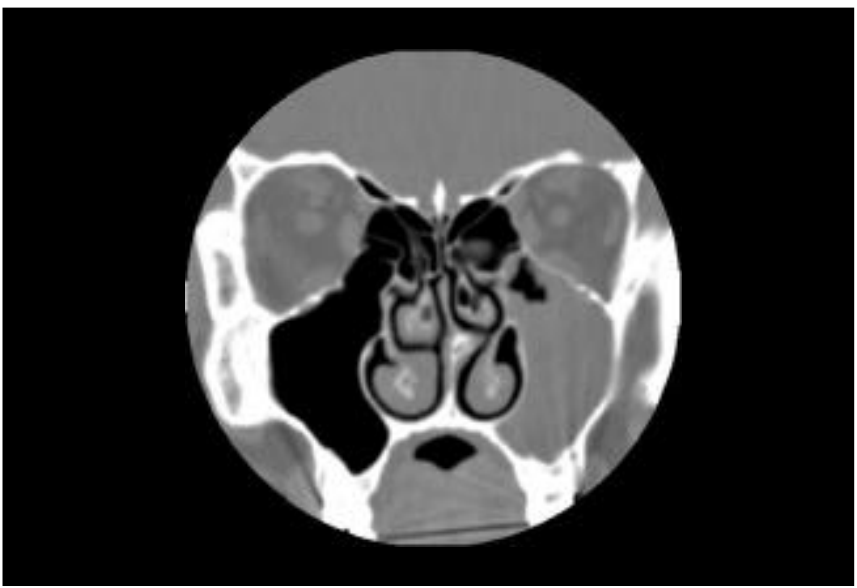

Figura 3. Corte coronal com desvio de septo para esquerda e sinusopatia maxilar esquerda

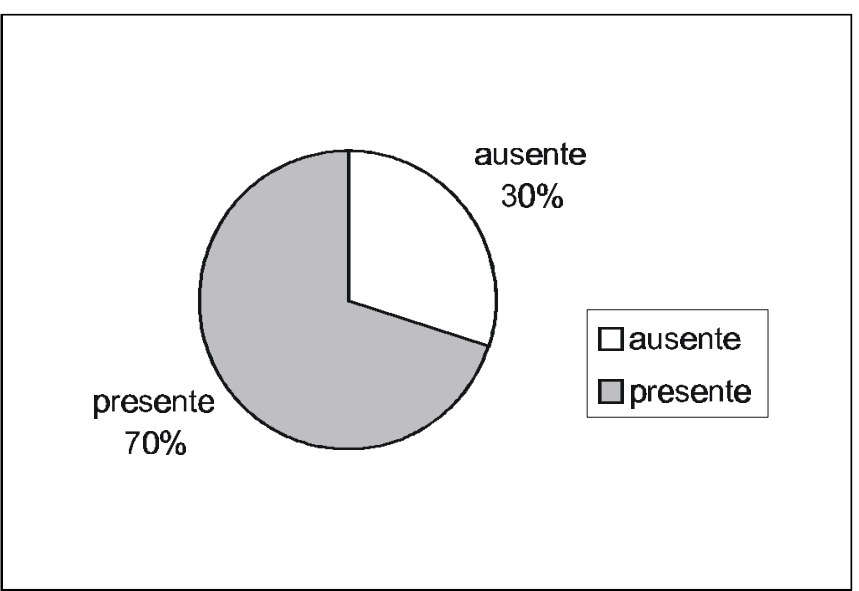

Gráfico 1. Distribuição de sinusopatias em tomografias computadorizadas de seios paranasais 


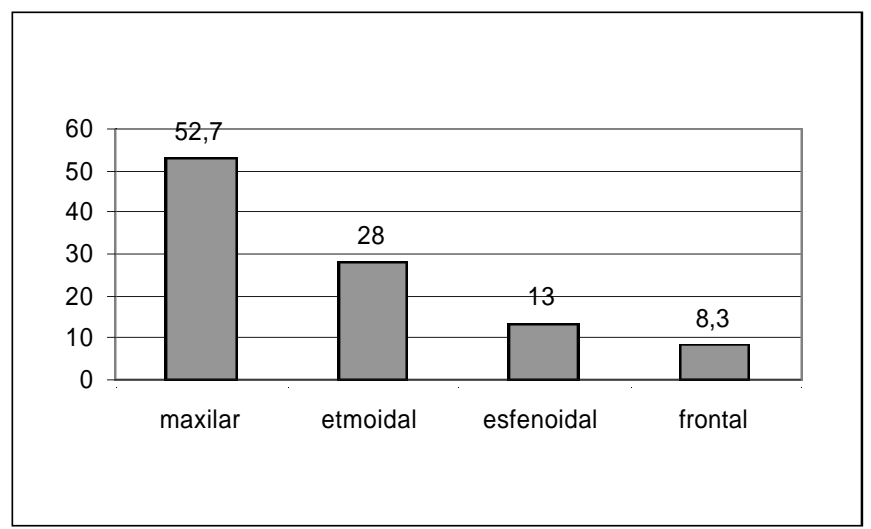

Gráfico 2. Distribuição dos seios paranasais mais acometidos

Tabela 1. Distribuição de sinusopatia maxilar e presença de célula de Haller ipslateral

\begin{tabular}{lcccccc}
\hline & \multicolumn{4}{c}{ Células de Haller } & \multicolumn{2}{c}{ Total } \\
Seio Maxilar & N & $\%$ & N & $\%$ & N & $\%$ \\
\hline Ausente & 129 & 47,4 & 13 & 46,4 & 142 & 47,3 \\
Presente & 143 & 52,6 & 15 & 53,6 & 158 & 52,7 \\
\hline Total & 272 & 100,0 & 28 & 100,0 & 300 & 100,0 \\
\hline
\end{tabular}

Teste: Qui-quadrado

Valor Observado: 0,00

Valor Crítico: 3,84

Tabela 2. Distribuição de sinusopatia etmoidal e presença de célula de Haller ipslateral

\begin{tabular}{lcccccc}
\hline & \multicolumn{4}{c}{ Células de Haller } & \multicolumn{2}{c}{ Total } \\
Seio etmoidal & $\mathrm{N}$ & $\%$ & $\mathrm{~N}$ & $\%$ & $\mathrm{~N}$ & $\%$ \\
\hline Ausente & 197 & 72,4 & 19 & 67,9 & 216 & 72,0 \\
Presente & 75 & 27,6 & 9 & 32,1 & 84 & 28,0 \\
\hline Total & 272 & 100,0 & 28 & 100,0 & 300 & 100,0 \\
\hline
\end{tabular}

Teste: Qui-quadrado

Valor Observado: 0,09

Valor Crítico: 3,84

Tabela 3. Distribuição de sinusopatia frontal e presença de célula de Haller ipslateral

\begin{tabular}{lcccccc}
\hline & \multicolumn{4}{c}{ Células de Haller } & \multicolumn{2}{c}{ Total } \\
Seio frontal & $\mathrm{N}$ & $\%$ & $\mathrm{~N}$ & $\%$ & $\mathrm{~N}$ & $\%$ \\
\hline Ausente & 251 & 92,3 & 24 & 85,7 & 275 & 91,7 \\
Presente & 21 & 7,7 & 4 & 14,3 & 25 & 8,3 \\
\hline Total & 272 & 100,0 & 28 & 100,0 & 300 & 100,0 \\
\hline
\end{tabular}

Teste: Qui-quadrado

Valor Observado: 0,70

Valor Crítico: 3,84

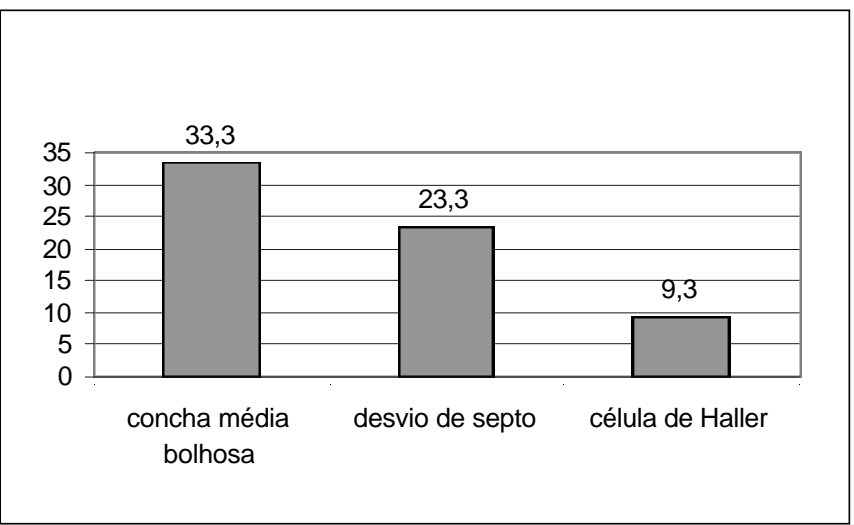

Gráfico 3. Distribuição das alterações anatômicas

Tabela 4. Distribuição de sinusopatia maxilar e presença de concha média bolhosa ipslateral

\begin{tabular}{lcccccc}
\hline & \multicolumn{4}{c}{ Concha Média Bolhosa } & \multicolumn{2}{c}{ Total } \\
Seio Maxilar & N & $\%$ & N & $\%$ & N & $\%$ \\
\hline Ausente & 89 & 44,5 & 53 & 53,0 & 142 & 47,3 \\
Presente & 111 & 55,5 & 47 & 47,0 & 158 & 52,7 \\
\hline Total & 200 & 100,0 & 100 & 100,0 & 300 & 100,0 \\
\hline Teste: Qui-quadrado & & & & & \\
Valor Observado: 1,61 & & & & & \\
Valor Crítico: 3,84
\end{tabular}

Tabela 5. Distribuição de sinusopatia etmoidal e presença de concha média bolhosa ipslateral

\begin{tabular}{lcccccc}
\hline & \multicolumn{4}{c}{ Concha Média Bolhosa } & \multicolumn{2}{c}{ Total } \\
Seio etmoidal & N & $\%$ & N & $\%$ & N & $\%$ \\
\hline Ausente & 138 & 69,0 & 78 & 78,0 & 216 & 72,0 \\
Presente & 62 & 31,0 & 22 & 22,0 & 84 & 28,0 \\
\hline Total & 200 & 100,0 & 100 & 100,0 & 300 & 100,0 \\
\hline
\end{tabular}

Teste: Qui-quadrado

Valor Observado: 2,25

Valor Crítico: 3,84

Tabelas 7, 8 e 9 também não mostram relação significante entre desvio de septo nasal com localização no meato médio e sinusopatia maxilar, etmoidal e frontal.

\section{DISCUSSÃO}

Variações nas células do agger nasi, conchas médias, processo uncinado, assim como a presença de células de Haller ou desvio do septo nasal, são citados como possíveis fatores etiológicos na doença sinusal e na cefaléia ${ }^{8}$. O utro fator que pode estar envolvido na etiologia das sinusites 
Tabela 6. Distribuição de sinusopatia frontal e presença de concha média bolhosa ipslateral

\begin{tabular}{lcccccc}
\hline & \multicolumn{4}{c}{ Concha Média Bolhosa } & \multicolumn{2}{c}{ Total } \\
Seio frontal & $\mathrm{N}$ & $\%$ & $\mathrm{~N}$ & $\%$ & $\mathrm{~N}$ & $\%$ \\
\hline Ausente & 180 & 90,0 & 95 & 95,0 & 275 & 91,7 \\
Presente & 20 & 10,0 & 5 & 5,0 & 25 & 8,3 \\
\hline Total & 200 & 100,0 & 100 & 100,0 & 300 & 100,0 \\
\hline
\end{tabular}

Teste: Qui-quadrado

Valor Observado: 1.58

Valor Crítico: 3,84

Tabela 7. Distribuição de sinusopatia maxilar e presença de desvio de septo ipslateral

\begin{tabular}{lccccccc}
\hline \multicolumn{9}{c}{ Mesmo lado sinusopatia de septo } & \multicolumn{2}{c}{ Ausente } & \multicolumn{2}{c}{ Total } \\
Seio Maxilar & $\mathrm{N}$ & $\%$ & $\mathrm{~N}$ & $\%$ & $\mathrm{~N}$ & $\%$ \\
\hline Ausente & 17 & 48,6 & 125 & 47,2 & 142 & 47,3 \\
Presente & 18 & 51,4 & 140 & 52,8 & 158 & 52,7 \\
\hline Total & 35 & 100,0 & 265 & 100,0 & 300 & 100,0 \\
\hline
\end{tabular}

Teste: Qui-quadrado

Valor Observado: 0,00

Valor Crítico: 3,84

inclui o contato entre as mucosas, que pode levar à perda da motilidade ciliar e à parada do transporte, provocando a estase de secreções, o que permite a infecção bacteriana ${ }^{3,8}$.

As variações anatômicas podem causar estenose adicional na parede nasal lateral, podendo impedir a drenagem e a ventilação dos seios ou causar um bloqueio. As variações podem, entretanto, ser assintomáticas e somente causar um bloqueio durante uma infecção viral ou bacteriana, devido a um edema da mucosa?.

O s estudos que relacionam variações anatômicas à ocorrência de sinusites têm sido controversos. Alguns autores afirmam que não há associação entre variações anatômicas e sinusite ${ }^{10}$. Vários destes estudos foram realizados apenas em populações de pacientes sintomáticos.

O utros autores compararam pacientes sintomáticos (ou sinusais) e assintomáticos (ou não sinusais). Foram considerados pacientes assinto máticos aqueles encaminhados para estudo tomográfico por outros motivos, geralmente para realizarem tomografia computadorizada de órbitas ${ }^{11,12}$. Neste modelo de estudo, existe uma limitação ao realizar-se a comparação entre tomografia computadorizada de seios paranasais e de órbitas. Já estão bem caracterizados os parâmetros técnicos a serem utilizados nos exames de seios paranasais, como a quilovoltagem, miliamperagem, tempo de exposição, algorítmo, plano, espessura e espaçamento dos cortes ${ }^{13}$. Na tomografia computadorizada de órbitas, to-
Tabela 8. Distribuição de sinusopatia etmoidal e presença de desvio de septo ipslateral

\begin{tabular}{lccccccc}
\hline \multicolumn{9}{c}{ Mesvio de septo } & \multicolumn{2}{c}{ Total } \\
Seio Etmoidal & $\mathrm{N}$ & $\%$ & $\mathrm{~N}$ & $\%$ & $\mathrm{~N}$ & $\%$ \\
\hline Ausente & 24 & 68,6 & 192 & 72,5 & 216 & 72,0 \\
Presente & 11 & 31,4 & 73 & 27,5 & 84 & 28,0 \\
\hline Total & 35 & 100,0 & 265 & 100,0 & 300 & 100,0 \\
\hline
\end{tabular}

Teste: Qui-quadrado

Valor Observado: 0,08

Valor Crítico: 3,84

Tabela 9. Distribuição de sinusopatia frontal e presença de desvio de septo ipslateral

\begin{tabular}{lccccccc}
\hline \multicolumn{7}{c}{ Mesvio de septo } & \multicolumn{2}{c}{ Total } \\
\multicolumn{1}{c}{ Seio frontal } & $\mathrm{N}$ & $\%$ & $\mathrm{~N}$ & $\%$ & $\mathrm{~N}$ & $\%$ \\
\hline Ausente & 32 & 91,4 & 243 & 91,7 & 275 & 91,7 \\
Presente & 3 & 8,6 & 22 & 8,3 & 25 & 8,3 \\
\hline Total & 35 & 100,0 & 265 & 100,0 & 300 & 100,0 \\
\hline
\end{tabular}

Teste: Qui-quadrado

Valor Observado: 0,00

Valor Crítico: 3,84

dos estes parâmetros técnicos são diversos, sendo apenas coincidentes os planos de corte. Além disso, vários trabalhos têm demonstrado a ocorrência de espessamento mucoso em indivíduos assintomáticos do ponto de vista nasosinusa ${ }^{14,15,16}$. Em vista disso, consideramos inadequada a utilização destes pacientes como grupo controle.

Em nossa opinião, a seleção ideal de pacientes assintomáticos deveria ser realizada a partir de indivíduos saudáveis, cuja normalidade naso-sinusal fosse constatada por exame clínico otorrinolaringológico, e que fosse aplicado a eles o protocolo de tomografia computadorizada de seios paranasais estabelecido pela literatura. Desta maneira, seria possível estabelecer a real incidência de espessamento mucoso e das variações anatômicas na população normal. Entretanto, a exposição de pessoas sadias à radiação ionizante levanta uma questão ética. Por esta razão, optamos apenas pelo estudo dos pacientes sintomáticos, analisando as diferenças intrínsecas deste grupo.

Em nosso estudo, observamos sinusopatia em $70 \%$ dos pacientes (Gráfico 1), confirmando a expectativa da literatura pesquisada. Bolger et al. ${ }^{14}$ verificaram doença mucosa em seios paranasais em $92,2 \%$ dos pacientes sinusais, Lloyd et al. ${ }^{17}$ observaram em $88 \%$ dos pacientes, Calhoun et al. ${ }^{18} \mathrm{em} 62 \%$, Tonai, Baba ${ }^{12} \mathrm{em} 93 \%$, Stackpole, Edestein ${ }^{19}$ em $58,4 \%$ e Kayalioglu et al. ${ }^{11}$ em $87,77 \%$.

Os seios paranasais mais acometidos, em ordem 
decrescente de freqüência foram (Gráfico 2): maxilares $(52,7 \%)$, etmoidais $(18,0 \%)$, esfenoidais $(13,0 \%)$ e frontais (8,3\%). Nossos achados, com relação à ordem de freqüência, assemelham-se aos de Calhoun et al. ${ }^{18}$ e Stackpole, Edelstein ${ }^{19}$. Os achados de Lloyd et al. ${ }^{17}$ só diferem no que se refere aos dois últimos seios afetados, pois estes autores encontraram maior incidência para os seios frontais do que para os esfenoidais. Zinreich et al. ${ }^{1}$ e Bolger et al. ${ }^{14}$ encontraram, em seus estudos, os seios etmoidais mais freqüentemente acometidos, seguidos pelos seios maxilares.

Zinreich et al. ${ }^{1}$ encontraram espessamento mucoso em $72 \%$ das tomografias computadorizadas em seio etmoidal anterior, $65 \%$ em seio maxilar, $34 \%$ em seio frontal, $40 \% \mathrm{em}$ seio etmoidal posterior e $29 \%$ em seio esfenoidal.

Bolger et al. ${ }^{14}$ verificaram doença mucosa em seios paranasais em $92,2 \%$ dos pacientes sinusais. Nestes pacientes, a região etmoidal anterior foi a mais comprometida $(84,3 \%)$, seguida pela maxilar $(77,7 \%)$, frontal $(36,6 \%)$, etmoidal posterior (38,6\%), e esfenoidal $(25,4 \%)$.

Calhoun et al. ${ }^{18}$ observaram anormalidade sinusal em $62 \%$ dos pacientes, sendo $43 \%$ em seio maxilar, $34 \%$ em células etmoidais anteriores, $21 \%$ em células etmoidais posteriores, $19 \%$ em seio esfenoidal e $13 \%$ em seio frontal.

Lloyd et al. ${ }^{17}$ encontraram $88 \%$ dos pacientes com alguma opacidade dos seios: em $83 \%$ o antro maxilar era acometido; o etmóide em $70 \%$ (63\% anterior e 57\% posterior); o seio frontal em $60 \%$; e o seio esfenoidal em $49 \%$.

Stackpole, Edelstein ${ }^{19}$ observaram evidência mucosa de sinusite em $58,4 \%$ dos pacientes. Sinusite maxilar foi observada em $47,4 \%$ de todos os pacientes, sinusite etmoidal em $35,7 \%$, e sinusite frontal ou esfenoidal em $19,5 \%$.

A incidência de células de Haller encontrada no presente trabalho foi de $9,3 \%$ (Gráfico 3). Os dados da literatura são muito discordantes, variando de $3,65 \%$ a $45,9 \% 10,11,12,14,17,19,20,21,22,23,24$. Como todos estes autores adotaram a mesma definição para célula de Haller, esta variação pode corresponder a diferenças intrínsecas das populações estudadas ou diferenças na sensibilidade de detecção dos métodos utilizados. Entretanto, em alguns estudos a incidência de células de Haller foi muito alta, 0 que nos leva a pensar que os autores podem ter incluído na casuística bolha etmoidal hiperpneumatizada, devido a erro durante a interpretação do exame.

A célula de Haller é uma expansão das células etmoidais que, em virtude de sua localização, pode potencialmente obstruir o óstio do seio maxilar e 0 infundíbulo etmoidal, causando rinossinusite. As Tabelas 1, 2 e 3 mostram que não há relação significante entre a presença de célula de Haller e sinusopatia maxilar, etmoidal e frontal.

Earwaker ${ }^{21}$ encontrou $20 \%$ de células de Haller e sugeriu que tal variação anatômica poderia ser um fator etiológico significativo na sinusite maxilar recorrente.
Stackpole, Edelstein ${ }^{19}$, analisando 154 pacientes com sintomas sinusais, observaram uma incidência de $34,4 \%$ de células de Haller. A células foram graduadas em pequenas, médias e grandes. Um aumento estatisticamente significante em doença mucosa do seio maxilar foi observado em pacientes com células de Haller de tamanho médio ou grande $(45,8 \%)$ se comparado às de tamanho pequeno $(28,9 \%)$.

A grande maioria dos autores não encontrou relação entre células de $\mathrm{Haller}$ e doença sinusal ${ }^{12,14,17}$. De qualquer forma, deve-se avaliar criteriosamente cada paciente, observando a dimensão das células de Haller, a presença de doença mucosa em seu interior e de contato mucoso.

0 diagnóstico da concha média bolhosa é mais bem realizado radiologicamente, uma vez que é facilmente visibilizada pela tomografia computadorizada ${ }^{1}$. A incidência de concha média bolhosa no presente trabalho foi de $33,3 \%$ (Gráfico 3). Esta distribuição encontra-se em concordância com a literatura, que freqüentemente se refere a uma incidência variando de $21 \%$ a $55 \%$ 1,10,11,12,14,17,18,21,22,25,26.

A diferença na incidência de concha média bolhosa verificada na literatura pode ser devido a fatores como diferenças intrínsecas das populações estudadas, nos critérios diagnósticos e na sensibilidade dos métodos de detecção. Estudos iniciais, por meio de dissecções anatômicas, indicam uma baixa incidência de concha média bolhosa, entre $8 \%$ a $20 \%$. Com o advento dos exames por imagem, mais precisamente a tomografia computadorizada, ocorreu uma melhora e um aumento no diagnóstico da concha média bolhosa.

As definições de concha média bolhosa também variam. Alguns autores só a consideram quando há pneumatização extensa, com aumento de volume ${ }^{7}$; outros definem como concha média bolhosa apenas o tipo bulbar? ${ }^{27}$. A literatura recente, entretanto, define como concha média bolhosa a presença de qualquer grau de pneumatização, subdividindo-a em lamelar, bulbar e extensa.

Por fim, há referências de diferenças raciais, como a menor incidência de concha média bolhosa na população japonesa. Este estudo também descreve que, quando presente, o grau de pneumatização seria pequeno ${ }^{12}$.

As Tabelas 4, 5 e 6 mostram a distribuição da concha média bolhosa e a presença de sinusopatia maxilar, etmoidal e frontal, e não se verifica relação significante entre as variáveis.

Y ousem et al.$^{26}$ encontraram uma incidência de $33 \%$ de pacientes com concha média bolhosa. Observaram pequena coincidência entre concha média bolhosa e sinusite maxilar e etmoidal (23 - 24\%), sendo mais importante 0 tamanho da concha média bolhosa que sua simples presença.

Lloyd et al. ${ }^{17}$ encontraram uma incidência de $24 \%$ de concha média bolhosa e não observaram relação com aumento de opacidade dos seios paranasais. Scribano et al. ${ }^{24}$ também não observaram relação entre concha média bolhosa e doença sinusal. 
Calhoun et al. ${ }^{18}$ observaram que a concha bolhosa era mais comum em pacientes com doença sinusal $(29,0 \%)$ que em pacientes não sinusais (15,9\%). Verificaram, ainda, que a presença de concha média bolhosa estava associada com doença etmoidal anterior, mas não com doença do complexo óstio-meatal.

Nadas et al. ${ }^{25}$ encontraram concha média bolhosa em $53 \%$ dos pacientes avaliados pela tomografia computadorizada de seios paranasais e observaram que a concha média bolhosa não era um fator significativo na gênese de anormalidades dos seios paranasais ou do complexo óstio-meatal.

Tonai, Baba ${ }^{12}$ encontraram $28,1 \%$ de concha média bolhosa no grupo sinusal, e $27,8 \%$ no grupo não sinusal. Relataram que a pneumatização da concha média pareceria não ser um fator muito importante na sinusite recorrente, uma vez que tal estrutura dificilmente desenvolver-se-ia a ponto de obstruir o meato médio.

No presente trabalho, encontramos desvio do septo nasal em 23,3\% (Gráfico 3) Também não encontramos relação significante entre presença de desvio do septo nasal e sinusopatia maxilar, etmoidal e frontal (Tabelas 7, 8 e 9). Blaungrund ${ }^{28}$ relatou uma incidência de desvio septal de $20 \%$. Earwaker ${ }^{21}$ encontrou desvio de septo em $44 \%$ dos casos. Yousem et al. ${ }^{26}$, analisando 100 tomografias de seios paranasais, observaram que pacientes com opacificação de um seio paranasal apresentavam desvio do septo nasal mais acentuado; entretanto, a presença de desvio de septo isoladamente não provocaria aumento da incidência de sinusopatia. Kayalioglu et al. ${ }^{11}$ observaram desvio do septo nasal em $22,22 \%$ dos pacientes com sintomas sinusais e em $12 \%$ dos pacientes sem sinto mas sinusais.

Calhoun et al. ${ }^{18}$ observaram que o desvio septal era mais comum nos pacientes sinusais $(40,0 \%)$ que nos pacientes não sinusais (19,5\%). A presença de um desvio septal foi significativamente associada com doença do complexo óstio-meatal, doença etmoidal anterior e doença etmoidal posterior.

Elahi et al. ${ }^{29}$ observaram relação entre doença do complexo óstio-meatal e desvio do septo nasal. Mais ainda, observaram um padrão entre ângulos aumentados de desvio septal e severidade de doença sinusal.

A diferença nos valores encontrados para a incidência do desvio de septo nasal pode ser explicada pelos critérios de avaliação de tal variação anatômica quanto ao seu grau e à sua localização. Neste trabalho, consideramos apenas desvios de septo nasal localizados no meato médio.

\section{CONCLUSÕES}

Neste estudo, após avaliação de 150 tomografias computadorizadas de seios paranasais, podemos concluir que:

1. Em pacientes com sintomas clínicos de sinusite, submetidos à tomografia computadorizada de seios paranasais, os seios mais freqüentemente acometidos são, em ordem decrescente: maxilar, etmoidal, esfenoidal e frontal.

2. A presença de concha média bolhosa, células de Haller ou desvio de septo nasal para o meato médio não está relacionada com sinusopatia maxilar, etmoidal e frontal, de maneira estatisticamente significante. Provavelmente, variações intrínsecas da mucosa nasal e paranasal podem influir de forma muito mais importante que as variações do complexo óstio-meatal.

\section{REFERÊNCIASBIBUOGRÁFICAS}

1. Zinreich SJ, Mattox DE, Kennedy DW, Chiisholm HL, Diffley DM, Rosenbaum AE. Concha bullosa: CT evaluation. J Comput Assist Tomogr 1988;12:778-84

2. Kennedy DW, Zinreich SJ, Rosenbaum AE, Johns ME. Functional endoscopic sinus surgery: theory and diagnostic evaluation. Arch Otolaryngol 1985;111:576-82.

3. Messerklinger $W$. On the drainage of the normal frontal sinus of man. Acta Otolaryngol 1967;63:176-81.

4. Stammberger H. Endoscopic endonasal surgery - concepts in treatment of rercurring rhinosinusitis. Part I. Anatomic and pathophysiologic considerations. Otolaryngol Head Neck Surg 1986;94(2):143-47

5. Zinreich SJ, Kennedy DW, Rosenbaum AE, Gayler BW, Kumar AJ, Stammberger $\mathrm{H}$. Paranasal sinuses: $\mathrm{CT}$ imaging requirements for endoscopic surgery. Radiology 1987;163:769-75.

6. Navarro JAC. Cavidade do nariz e seios paranasais. Bauru: All Dent; 1997. $160 \mathrm{p}$.

7. Som PM. CT of the paranasal sinuses. Neuroradiology $1985 ; 27: 189-$ 201.

8. Stammberger $\mathrm{H}$, Wolf $\mathrm{G}$. Headaches and sinus disease: the endoscopic approach. Ann Otol Rhinol Laryngol 1988;97(134):323.

9. Kopp W, Stammberger H, Fotter R. Special radiologic imaging of paranasal sinuses. Eur J Radiol 1988;8:153-6.

10. Ünlü HH, Akyar S, Çaylan R, Nalça Y. Concha bullosa. J Otolaryngol 1994;23(1):23-7.

11. Kayalioglu G, Oyar O, Govsa F. Nasal cavity and paranasal sinus bony variations: a computed tomographic study. Rhinology 2000;38(3):108-13.

12. Tonai $\mathrm{A}, \mathrm{Baba} \mathrm{S}$. Anatomic variations of the bone in sinonasal $\mathrm{CT}$. Acta Otolaryngol Suppl (Stockh) 1996;525:9-13.

13. Babbel R, Harnsberger HR, Nelson B, Solkens J, Hunt $S$. Optimization of techniques in screening $C T$ of the sinuses. AJR 1991;157:1093-98.

14. Bolger WE, Butzin CA, Parsons DS. Paranasal sinus bony anatomic variations and mucosal abnormalities: CT analysis for endoscopic sinus surgery. Laryngoscope 1991;101:56-64.

15. Diament MJ, Senac MO Jr, Gilsanz V, Baker S, Gillespie T, Larsson $S$. Prevalence of incidental paranasal sinuses opacification in pediatric patients: a CT study. J Comput Assist Tomogr 1987;11(3):426-31.

16. Havas TE, Motbey JA, Gullane PJ. Prevalence of incidental abnormalities on computed tomographic scans of the paranasal sinuses. Arch Otolaryngol Head Neck Surg 1988;114:856-9.

17. Lloyd GAS, Lund VJ, Scadding GK. CT of the paranasal sinuses and functional endoscopic surgery: a critical analysis of 100 symptomatic patients. J Laryngol Otol 1991;105:181-5.

18. Calhoun KH, Waggenspack GA, Simpson CB, Hokanson JA, Bailey BJ. CT evaluation of the paranasal sinuses in symptomatic and asymptomatic populations. Otolaryngol Head Neck Surg $1991 ; 104(4): 480-3$. 
19. Stackpole AS, Edelstein DR. The anatomic relevance of the Haller cell in sinusitis. Am J Rhinol 1997;11(3):219-23.

20. Danese M, Duvoisin B, Agrifoglio A, Cherpillod J, Krayenbuhl M. Influence des variantes anatomiques nasosinusales sur les sinusites récidivantes, persistantes ou chroniques. Évaluation TDM chez 112 patients. J Radiol 1997;78(9):651-7.

21. Earwaker J. Anatomic variants in sinonasal CT. RadioGraphics 1993;13:381-415.

22. Meloni F, Rovasio S, Stomeo F, Teatini GP. Anatomic variations of surgical importance in ethmoid labyrinth and sphenoid sinus. A study of radiological anatomy. Surg Radiol Anat 1992;14:65-70.

23. Milczuk HA, Dalley RW, Wessbacher FW, Richardson MA. Nasal and paranasal sinus anomalies in children with chronic sinusitis. Laryngoscope 1993;103(3):247-52.

24. Scribano E, Ascenti G, Loria G, Cascio F, Gaeta M. The role of the ostiomeatal unit anatomic variations in inflamatory disease of the maxillary sinuses. Eur J Radiol 1997;24(3):172-4.

25. Nadas S, Duvoisin B, Landry M, Schnyder P. Concha bullosa: frequency and appearances on $C T$ and correlations with sinus disease in 308 patients with chronic sinusitis. Neuroradiology $1995 ; 37(3): 234-7$.
26. Yousem DM, Kennedy DW, Rosenberg S. Ostiomeatal complex risk factors for sinusitis: CT evaluation. J Otolaryngol 1991;20:41924

27. Ferrie JC, Azais O, Vandermarcq P, Klossek JM, Drouineau J, Gasquet C. Exploration tomodensitométrique de l'ethmoïde et du méat moyen. II. Radio-anatomie (incidence axiale) et variations morphologiques. J Radiol 1991;72(10):477-87.

28. Blaugrund SM. The nasal septum and concha bullosa. Otolaryngol Clin North Am 1989;22:291-306.

29. Elahi MM, Frenkiel S, Fageeh N. Paraseptal structural changes and chronic sinus disease in relation to the deviated septum. J Otolaryngol 1997;26(4):236-40.

30. Basic N, Basic V, Jukic T, Basic M, Jelic M, Hat J. Computed tomographic imaging to determine the frequecy of anatomical variations in pneumatization of the ethmoid bone. Eur Arch Otorhinolaryngol 1999;256(2):69-71. 